\title{
Transcriptional control of several aerobically induced cytochrome structural genes in Rhodobacter sphaeroides
}

\author{
Janice E. Flory ${ }^{1}$ and Timothy J. Donohue ${ }^{1,2}$
}

Graduate Program in Cell and Molecular Biology' and Department of Bacteriology², University of Wisconsin-Madison, Madison, WI 53706, USA
Author for correspondence: Timothy J. Donohue. Tel: +1 608262 4663. Fax: +1 6082629865 . e-mail: tdonohue@bact.wisc.edu

To decipher how the synthesis of energy-transducing enzymes responds to environmental cues, the response of three Rhodobacter sphaeroides aerobic cytochrome gene promoters was analysed under different conditions. Two of these promoters are upstream of structural genes (ctaD and coxII) for individual subunits of the cytochrome $\mathrm{aa}_{3}$ respiratory complex. The third promoter is that for the CycFG operon, which encodes two c-type cytochromes

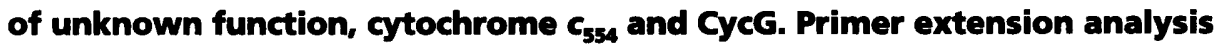
identified a single oxygen-responsive transcription start site for each gene. Utilizing operon fusions to Escherichia coli lacz as a measure of promoter activity, transcription from the ctaD, coxll and cycFG promoters was approximately twofold higher when cells were grown at high (30\%) oxygen tensions than under low (2\%) oxygen or anaerobic (photosynthetic) conditions. Analysis of promoter function using specific host mutations indicated that loss of the $R$. sphaeroides FNR homologue, FnrL, causes a small, but reproducible, increase in cycFG and coxll transcription when cells are grown at $2 \%$ oxygen. However, neither the $\Delta F n r L$ mutation nor alterations in sequences related to a consensus target site for the $E$. coli FNR protein increased function of any of these three promoters to that seen under aerobic conditions in wild-type cells. From this we conclude that FnrL is not solely responsible for reduced transcription of these three aerobic cytochrome genes under low oxygen or anaerobic conditions. When activity of these three promoters was monitored after cells were shifted from anaerobic (photosynthetic) conditions to a $\mathbf{3 0} \%$ oxygen atmosphere, it took several cell doublings for Lacz levels to increase to those found in steady-state $30 \%$ oxygen cultures. From these results, it appears that activity of these promoters is also regulated by a stable molecule whose synthesis or function responds slowly to the presence of high oxygen tensions.

Keywords: cytochromes, gene expression, transcriptional control, anaerobic regulation, oxygen regulation

\section{INTRODUCTION}

Most facultative bacteria contain multiple energygenerating pathways whose synthesis is often controlled by overlapping global regulatory networks. By coordinating expression of specific respiratory components, these networks help direct electron flow through particular energy-generating pathways under

Abbreviations: $\mathrm{cyt} \mathrm{aa}_{3}$, cytochrome $\mathrm{aa}_{3}$ oxidase; LacZ, $\beta$-galactosidase. different environmental conditions. This study sought to define metabolic and genetic elements that control expression of cytochromes known to either function or to be induced under aerobic respiratory conditions in the facultative phototroph Rhodobacter sphaeroides. While genes encoding components of the $R$. sphaeroides photosynthetic apparatus are known to respond to oxygen, light and tetrapyrrole availability (Lee \& Kaplan, 1992; Schilke \& Donohue, 1992; Eraso \& Kaplan, 1994; Penfold \& Pemberton, 1994; Gomelsky $\&$ Kaplan, 1995), factors governing expression of 
aerobic electron carriers in this facultative phototroph have received relatively little attention.

Like most bacterial species, $R$. sphaeroides contains a branched aerobic respiratory chain (García-Horsman et al., 1994a). The cytochrome $a a_{3}$ oxidase (cyt $a a_{3}$ ) of this bacterium is a well-characterized prokaryotic homologue of the mitochondrial cytochrome oxidase and a member of the haem-copper oxidase superfamily (Cao et al., 1991, 1992; Shapleigh \& Gennis, 1992). From studies on many haem-copper oxidases, a paradigm has developed in which the cyt $a a_{3}$ family members are maximally expressed in highly aerated bacteria because they have a lower affinity for oxygen than most of the alternative cytochrome oxidases (García-Horsman et al., 1994a ; Trumpower \& Gennis, 1994). To test if such differential expression of aerobic cytochromes occurs in $R$. sphaeroides, we examined transcription of two cyt $a a_{3}$ genes (ctaD and coxII), both in steady-state cultures grown at different oxygen tensions and when photosynthetic (anaerobic) cultures were shifted to high $(30 \%)$ oxygen conditions. Since previous work suggested that the elevated levels of cytochrome $c_{554}$ found under high $(30 \%)$ oxygen growth conditions reflects increased cycFG transcription (Flory \& Donohue, 1995), we also analysed function of this promoter. In considering how these genes might be regulated by changes in oxygen tension, the existence of sites similar to the consensus sequence for the Escherichia coli global anaerobic regulator FNR in the cycFG, coxIl and ctaD control regions (Cao et al., 1991; Shapleigh \& Gennis, 1992; Flory \& Donohue, 1995) make it possible that their expression is repressed under anaerobic conditions by the $R$. sphaeroides FNR homologue, FnrL (ZeilstraRyalls \& Kaplan, 1995). All three of these promoters are oxygen-regulated since $\beta$-galactosidase (LacZ) levels from specific operon fusions to $E$. coli lac $Z$ are reduced under low oxygen or anaerobic (photosynthetic) growth conditions. However, by monitoring promoter function from mutant reporter genes either in wild-type cells or in a defined FnrL null mutant it appears that this $R$. sphaeroides global anaerobic transcription factor is not solely responsible for normal oxygen control of their transcription. Indeed, the time-dependent increases in promoter activity when oxygen is added to anaerobic (photosynthetic) cultures suggest that $c y c F G$, coxII and ctaD transcription is also controlled by a stable, anaerobic repressor or an activator that is synthesized during growth in the presence of high $(30 \%)$ oxygen.

\section{METHODS}

Cell growth, media and genetic techniques. $R$. sphaeroides cells (Table 1 ) were grown on solid media or in liquid cultures of Sistrom's minimal medium A at $32^{\circ} \mathrm{C}$ (Donohue et al., 1986). Aerobic cultures $(100 \mathrm{ml})$ were sparged either with a gas mixture known to repress photosynthetic membrane synthesis $\left(30 \% \mathrm{O}_{2}, 69 \% \mathrm{~N}_{2}, 1 \% \mathrm{CO}_{2}\right.$; Chory et al., 1984) or at low oxygen tensions $\left(2 \% \mathrm{O}_{2}, 97 \% \mathrm{~N}_{2}, 1 \% \mathrm{CO}_{2}\right)$. Steady-state anaerobic (photosynthetic) cells were grown in filled tubes in front of a tungsten light bank (Donohue et al., 1986).

For shifting cultures from anaerobic (photosynthetic) to respiratory ( $30 \%$ oxygen) growth conditions, illuminated $500 \mathrm{ml}$ cultures were sparged for several doublings with $95 \%$ $\mathrm{N}_{2}$ and $5 \% \mathrm{CO}_{2}$. When indicated, the gas mixture was changed to $30 \% \mathrm{O}_{2}, 69 \% \mathrm{~N}_{2}, 1 \% \mathrm{CO}_{2}$ and illumination terminated. Cultures were diluted into fresh medium, as necessary, to maintain exponential growth during the course of the experiment.

$E$. coli cells were grown in LB on a rotary shaker or on solid media at $37^{\circ} \mathrm{C}$ (Sambrook et al., 1989). To maintain plasmids (Table 1) in E. coli, ampicillin $\left(50 \mu \mathrm{g} \mathrm{ml}^{-1}\right)$, kanamycin $\left(25 \mu \mathrm{g} \mathrm{ml}^{-1}\right)$, spectinomycin $\left(25 \mu \mathrm{g} \mathrm{m}^{-1}\right)$ or tetracycline $\left(10 \mu \mathrm{g} \mathrm{ml}^{-1}\right)$ were used. For $R$. sphaeroides, spectinomycin and kanamycin were used at $25 \mu \mathrm{g} \mathrm{ml}^{-1}$ and tetracycline was added to $1 \mu \mathrm{g} \mathrm{ml}^{-1}$. After diparental mating between E. coli S17-1 (Simon et al., 1983) and $R$. sphaeroides recipients (Donohue et al., 1988), cells were plated aerobically on media containing spectinomycin and kanamycin to select for exconjugants.

DNA manipulations, cloning and sequencing techniques. Restriction enzymes, T4 DNA ligase, T4 DNA polymerase and Klenow fragment DNA polymerase were used according to suppliers' specifications and standard techniques (Sambrook et al., 1989). Plasmid DNA was prepared with Qiagen kits; DNA was isolated from agarose gels using Prep A Gene kits (Bio-Rad). Taq DNA polymerase was used for dideoxy DNA sequencing with deazanucleotide triphosphates (Promega) and either vector (Stratagene) or $R$. sphaeroides-specific primers (Genosys Biotechnologies). Sequence analysis was aided by University of Wisconsin Genetics Computer Group software (Devereux et al., 1984).

Use of pRKK200 to construct lacZ operon fusions. Plasmid pRKK200 (R. K. Karls \& T. J. Donohue, unpublished) is a kanamycin-resistant pKT231 derivative (Bagdasarian \& Timmis, 1982) that contains a promoterless $E$. coli lac $Z$ gene downstream of a strong transcription terminator on the $\Omega S^{r}$ cassette (Prentki \& Krish, 1984). This plasmid has unique $K p n I$ and StuI sites for directional cloning of DNA fragments upstream of $l a c Z$. Similar IncQ plasmids exist at 4-10 copies per R. sphaeroides cell (Donohue \& Kaplan, 1991).

cycFG operon fusions. To construct a $c y c F G$ : : lac $Z$ fusion, a $140 \mathrm{bp} M s c I$ restriction fragment of $c y c F G$ promoter DNA (Flory \& Donohue, 1995) was cloned into the StuI site of pRKK200. DNA sequencing identified a plasmid in which the cycFG promoter was fused to lacZ (pRKKcycF).

For creating $c y c F G$ promoter mutations, the same DNA was cloned into the SmaI site of pUC119 (pTC119F) and transformed into RZ1032 (Kunkel, 1985). Single-stranded DNA (Kunkel, 1985) was hybridized to mutagenic oligonucleotides cycFFNR (CGATCACATTAAAGCT A ACATTTTTCTAGTATGTCTGTCG) to create a mutant FNR site (cycFG-1 allele), or cycFREP (CCAGTATGTCTGTCGTAGTTAGCGGGCTAGATTTC) to destroy an inverted repeat in the $c y c F G$ promoter spacer ( $c y c F G-2$ allele). After confirming individual mutations (indicated by underlining) by DNA sequencing, $K p n \mathrm{I}$ and HincII were used to isolate mutant cycFG promoter fragments for cloning into KpnI and StuIdigested pRKK200. This mutagenesis scheme added $2 \mathrm{bp}$ of upstream DNA and 17 bp before the start of lac $Z$ compared to the wild-type $c y c F G$ ::lacZ fusion. The additional downstream DNA accounts for the size difference in primer extension products from a wild-type $c y c F G$ : : lacZ fusion and those from analogous $c y c F G-1$ or $c y c F G-2$ reporter genes (see Results).

coxll operon fusions. A 282 bp coxII DNA fragment (Cao et al., 1991) was amplified by PCR using the primers coxIIPCR1 
Table 1. Bacterial strains and plasmids

\begin{tabular}{|c|c|c|}
\hline Designation & Relevant feature & Source or reference \\
\hline \multicolumn{3}{|l|}{ Strains } \\
\hline 2.4 .1 & R. sphaeroides, wild-type & Laboratory strain \\
\hline JZ1678 & R. sphaeroides, fnrL::kan & Zeilstra-Ryalls \& Kaplan (1995) \\
\hline RZ1032 & E. coli, dut ung & Kunkel (1985) \\
\hline S17-1 & E. coli, $\mathrm{mob}^{+}$ & Simon et al. (1983) \\
\hline \multicolumn{3}{|l|}{ Plasmids } \\
\hline pRKK200 & Promoterless lacZ plasmid & R. K. Karls (this laboratory) \\
\hline pRKKcycF & $\mathrm{pRKK} 200+c y c F G \mathrm{p}$ & This work \\
\hline pRKKF-FNR & pRKK200+cycFGp FNR site mutant (cycFG-1) & This work \\
\hline pRKKF-REP & pRKK $200+c y c F G$ p spacer mutant $(c y c F G-2)$ & This work \\
\hline pRKKctaD & $\mathrm{pRKK} 200+c t a D \mathrm{p}$ & This work \\
\hline pRKKcoxII & $\mathrm{pRKK} 200+\operatorname{coxIIp}$ & This work \\
\hline pRKKIIFNR & pRKK $200+\operatorname{coxIIp~FNR~site~mutant~(coxIl-1)~}$ & This work \\
\hline pUC119 & Phagemid vector & Clonetech \\
\hline pTC119F & $\mathrm{pUC} 119+c y c F G \mathrm{p}$ & This work \\
\hline pTC119FNR & pUC119+cycFGp FNR site mutant (cycFG-1) & This work \\
\hline pTC119REP & pUC119+cycFGp spacer mutant $(c y c F G-2)$ & This work \\
\hline pBSIIKS - & Phagemid vector & Stratagene \\
\hline pBScoxII & pBSIIKS-+ coxIIp & This work \\
\hline pBSIIFNRII & pBSIIKS- + coxIlp FNR site mutant (coxIl-1) & This work \\
\hline pJS3 & pT3T718 $+c t a D$ coding sequence & Shapleigh \& Gennis (1992) \\
\hline
\end{tabular}

(GCTGGTACCCTCGGAGGCATCGTAAC) and coxIIPCR2 (AGAGTCCGTCGACCCGTTGTTCCTGAACCG). The $\sim 300$ bp product was digested with KpnI and HincII (underlined), ligated into KpnI and StuI-digested pRKK200, and sequenced to identify a wild-type coxII::lacZ reporter plasmid (pRKKcoxII).

For mutagenesis, this PCR product was cloned into $K p n I$ and HincII-digested pBSIIKS -. After finding a plasmid where no mutations were introduced into the coxII promoter by PCR (pBScoxII), the Fnr site was mutagenized (Kunkel, 1985) with the coxFNR primer (CATGACTACTGTCCCTAGTTCAGACTAGATGATGACGCTTCG). A mutant coxII promoter fragment (coxII-1) was isolated by digestion with $K p n I$ and HincII and cloned into KpnI and StuI-digested pRKK200 to generate a coxII-1::lacZ reporter plasmid ( $\mathrm{pRKKIIFNR).}$

ctaD operon fusions. A 226 bp restriction fragment of the $c t a D$ upstream region was isolated as a SmaI-MscI fragment from pJS3 (Shapleigh \& Gennis, 1992) and ligated into the StuI site of pRKK200. DNA sequencing identified a plasmid with lacZ under $c t a D$ promoter control (pRKKctaD).

RNA techniques. Cellular RNA was prepared (Zhu \& Kaplan, 1985), quantified (Donohue et al., 1986) and used in primer extension assays (Karls et al., 1989) with avian myeloblastosis virus reverse transcriptase (Molecular Genetic Resources). Primers were: cycF10 (AGGCCGTGGCGCCTTCGAT), coxII-1 (AGGTCGTGGAATGTCTCATG), and ctaD-1 (CTGCGTCGGCCATTGGCTTG). Products were separated on denaturing urea/polyacrylamide gels alongside DNA sequencing ladders.

Lacz assays. $R$. sphaeroides cells containing individual transcriptional fusions were assayed in triplicate for LacZ activity (Schilke \& Donohue, 1992). Cells were harvested in the exponential phase of growth to minimize unwanted secondary effects on promoter activity. Data presented are the mean $\pm \mathrm{SD}$ of at least three independent cultures. The Student's $t$ test was used to assess if LacZ levels in individual cultures were significantly different from each other.

Bacteriochlorophyll analysis. For these assays, $25 \mathrm{ml}$ culture samples were harvested, stored at $-20^{\circ} \mathrm{C}$ and resuspended in $0.2 \mathrm{ml}$ deionized water to determine the specific bacteriochlorophyll content. Half of the sample was used for protein determination (Markwell et al., 1978); the remainder was assayed for bacteriochlorophyll (Cohen-Bazire et al., 1957).

\section{RESULTS AND DISCUSSION}

\section{Activity of the ctaD, coxll and cycFG promoters is increased under aerobic conditions}

By primer extension assays the start site for coxII transcription maps $80 \mathrm{nt}$ upstream of the initiator methionine, while that for $c t a D$ is $54 \mathrm{nt}$ upstream of the start of translation (summarized in Fig. 1). Each gene appears to be transcribed from a single promoter because the primer extension product is the same when RNA from aerobically or anaerobically grown cells is used (Fig. 2 for coxII, data not shown for $c t a D$ ). Both primer extension assays and Northern blot analysis suggested that the ctaD and coxII promoters were oxygen-regulated since there was more mRNA in $30 \%$ oxygen cells than in those grown under anaerobic (photosynthetic) conditions (see Fig. 2 for coxll primer extension assays, data not shown for $c t a D$ ).

To facilitate the study of oxygen-dependent control of $c t a D$, coxII and $c y c F G$ transcription, individual lacZ operon fusions were constructed. To verify that these low-copy reporter genes were valid indicators of pro- 


\section{CyCFG}

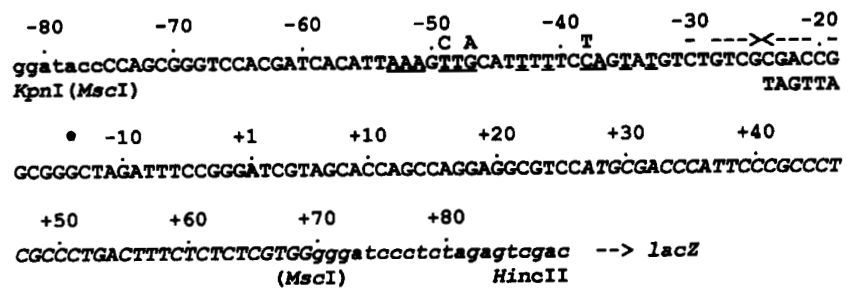

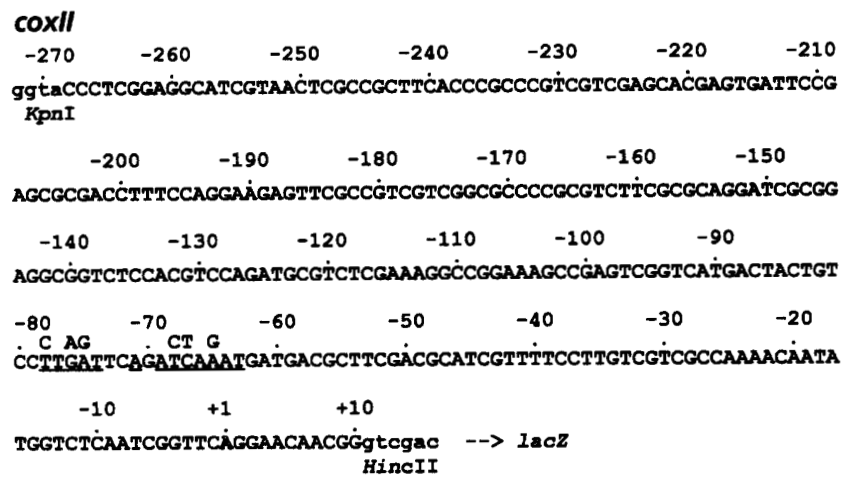

ctaD

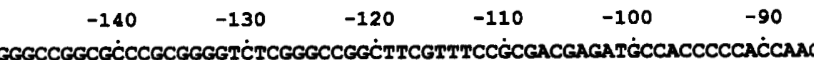
(SmaI)

$\begin{array}{llllll}-80 & -70 & -60 & -50 & -40 & -30\end{array}$

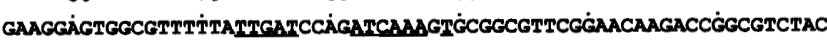
$\begin{array}{lllllll}-20 & -10 & +1 & +10 & +20 & +30 & +40\end{array}$

AGATATCCGGGÁGATGCTGGCÁCGGATCCAT́CCGACGCCC'́TCTGATCGGAAACAGCACGĆGGG $+50+60 \quad+70$

GAGGCAÁGCCAATGGCĆGACGCAGCCATCCATGG $-->1 \mathrm{acZ}$ (MscI)

\section{E. coli FNR consensus recognition site

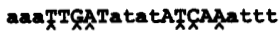

Fig. 1. Promoter sequences used to construct lac $Z$ operon fusions to CycFG (accession no. L36880; positions 245-388; Flory \& Donohue, 1995), coxll (M57680; 1-280; Cao et al., 1991) and ctaD (X62645; 4-229; Shapleigh \& Gennis, 1992). The transcription start sites $(+1$, outlined), potential FNR target sequences (underlined), mutations to generate mutant promoters (above the line) and the start of translation (italics) are indicated. Sequences present in the lac $Z$ operon fusions that are not found in their genomic counterparts (lower case letters) and endonuclease recognition sites destroyed during cloning (parentheses) are also shown. An inverted repeat in the cycFG sequence (arrows), the half-site substitution (below the line) and the position of an additional transcription start site in the cycFG-2 mutant promoter that resulted from this mutation (asterisk) are indicated. The consensus binding site for $E$. coli FNR and bases proposed to make site-specific protein contacts (carets) are presented at the bottom (Spiro et al., 1990; Lazazzera et al., 1993).

moter activity, primer extension assays showed that the lacZ-specific transcripts had the same $5^{\prime}$ end as their genomic counterparts (data not shown). Cells containing any of these three reporter genes produced LacZ activity well over the background observed from a control strain containing a promoterless lac $Z$ gene

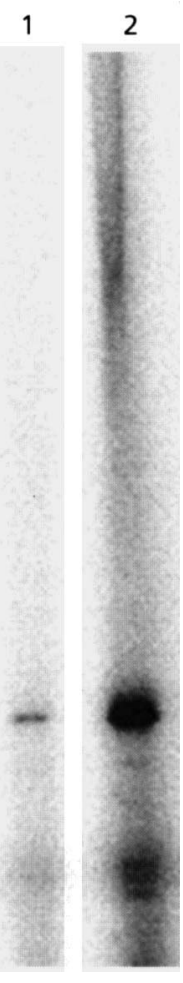

Fig. 2. coxll mRNA levels are increased under aerobic conditions. Genomic primer extension products using $40 \mu \mathrm{g}$ RNA from wild-type cells grown under anaerobic (photosynthetic) (lane 1) or $30 \%$ oxygen (lane 2 ) conditions are shown.

(pRKK200, <6 Miller units). In addition, LacZ levels in cells containing the $c t a D$, coxII or $c y c F G$ reporter genes were approximately twofold higher when cells were grown at high $(30 \%)$ oxygen levels rather than under anaerobic (photosynthetic) conditions (Fig. 3). This suggests that a portion of the increased mRNA abundance is due to elevated transcription. While a contribution of additional cis-acting sequences to promoter function is still possible, these data indicate that the amount of each promoter DNA which is fused to lacZ (268 bp for coxII, $149 \mathrm{bp}$ for $c t a D$ or $89 \mathrm{bp}$ for $c y c F G$ ) is sufficient to increase transcription at $30 \%$ oxygen.

To further evaluate the effects of oxygen tension on $c t a D$, coxII and $c y c F G$ expression, LacZ levels were measured in cells grown under a $2 \%$ oxygen atmosphere, a condition sufficient to induce photosynthetic membrane assembly (Cohen-Bazire et al., 1957). Activity of the $c t a D, c o x I l$ and $c y c F G$ promoters in cells grown in a $2 \%$ oxygen environment was similar to that found in anaerobic (photosynthetic) cultures (Fig. 3). Such a reduction in ctaD and coxII promoter function at reduced oxygen tensions was the response expected given the low affinity of cyt $a a_{3}$ complexes for oxygen (Trumpower \& Gennis, 1994). Indeed, the E. coli equivalent to cyt $a a_{3}$ (cytochrome $b o_{3}$ oxidase) is also transcribed less at low oxygen (Iuchi et al., 1990; Minagawa et al., 1990). Thus, these estimates of coxII 


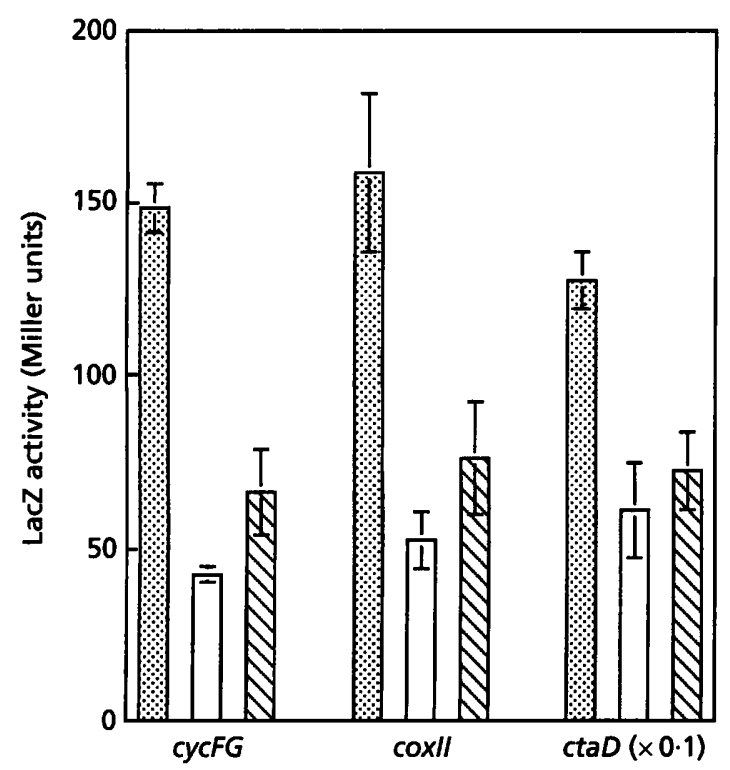

Fig. 3. LacZ specific activities of wild-type cells containing the indicated reporter genes in cells grown under $30 \%$ oxygen (图), $2 \%$ oxygen $(\square)$ or anaerobic (photosynthetic) $(\mathbb{})$ conditions. Reported values are the mean $\pm S D$ of at least three independent assays and are corrected for activity from the vector alone under the same conditions (pRKK200, <6 Miller units). Note that LacZ levels from the ctaD reporter fusion are reported at one-tenth of the actual values. Relevant reporter plasmids are as follows (Table 1): cycFG, pRKKcycF; coxll, pRKKCoxII; ctaD, pRKKctaD.

and $c t a D$ promoter function provide a provisional indication that cyt $a a_{3}$ levels are lower at reduced oxygen tensions. However, the modest reduction in ctaD and coxIl promoter activity at $2 \%$ oxygen or anaerobic conditions suggests these cells contain a considerable pool of cyt $a a_{3}$ complexes. If $R$. sphaeroides regulates synthesis of its high affinity oxidase(s) as in other bacteria (García-Horsman et al., 1994a, b) they should be maximally expressed at low oxygen tensions (Iuchi et al., 1990; Fu et al., 1991; Moshiri et al., 1991).

Given that $\mathrm{CtaD}$ and CoxII are found in equimolar amounts within the cyt $a a_{3}$ complex, we were surprised that $c t a D$ promoter activity was approximately ninefold higher than its coxII counterpart (Fig. 3). This difference could reflect deletion of important cis-acting sequences on either reporter gene. However, differences in stability of $c t a D$ and coxII transcripts could also explain both the apparent strengths of the coxII and $c t a D$ promoters and our difficulty in reproducibly obtaining ctaD primer extension products (see above). Alternatively, ctaD transcription could generate a stable pool of $\mathrm{CtaD}$ for assembly of the cyt $a a_{3}$ complex. A similar role as a membrane-bound nucleation site for newly synthesized enzyme complexes has been proposed for the $\mathrm{H}$ subunit of the R. sphaeroides reaction centre complex (Chory et al., 1984).

\section{Function of the aerobic cytochrome promoters increases slowly after exposure to $30 \%$ oxygen}

To examine how oxygen controls $c t a D$, coxII and $c y c F G$ promoter function, the kinetics of $\mathrm{LacZ}$ accumulation from individual reporter genes was monitored after photosynthetic cells were exposed to $30 \%$ oxygen (Fig. 4). Photosynthetically grown $R$. sphaeroides cells contain considerable cytochrome oxidase activity (GarcíaHorsman et al., 1994a, b), so it is not surprising that cell growth continues when these cells are exposed to $30 \%$ oxygen. When oxygen was introduced into steady-state anaerobic (photosynthetic) cultures, there was a slow, progressive increase in $\mathrm{LacZ}$ activity from each operon fusion. Indeed, about $24 \mathrm{~h}$ (six cell doublings) after the introduction of $30 \%$ oxygen were required for $\mathrm{LacZ}$ levels from each reporter fusion to increase to that found in steady-state cells grown under these conditions. The slow increase in activity of these three promoters seems to preclude function of either a pre-existing oxygen sensor or a rapidly metabolized intermediate as negative transcriptional regulators. Rather, the slow increase in transcription could reflect time required for an activator or co-inducer of $c t a D$, coxII and $c y c F G$ promoter function to accumulate after photosynthetic cells are shifted to a $30 \%$ oxygen atmosphere. Another equally plausible alternative is that $c t a D$, coxII and $c y c F G$ promoter function is repressed by an oxygen-insensitive transcription factor that uses a component of the photosynthetic apparatus or a stable compound that is accumulated under low oxygen conditions as a corepressor. In either of these two latter cases, coxIl, ctaD and $c y c F G$ promoter function would only increase after the putative repressor, pre-existing photosynthetic units, or a potential co-repressor was diluted by growth at $30 \%$ oxygen.

The rapid cessation of photosynthetic membrane synthesis that occurs when $R$. sphaeroides is exposed to high oxygen tensions (Cohen-Bazire et al., 1957) allowed us to test if the increased activity of these three promoters under these conditions could be correlated with dilution of a regulatory factor from photosynthetic cultures. When we assayed the specific bacteriochlorophyll content of these cells (as a marker for photosynthetic membrane content), we observed the expected time-dependent decrease in this parameter (Fig. 4) as pre-existing photosynthetic membranes were diluted by cell division after oxygen was introduced (Cohen-Bazire et al., 1957). When all the data are considered together, it appears that increased function of these three promoters parallels the loss of pre-existing components from photosynthetic cells.

\section{The $R$. sphaeroides FNR homologue, FnrL, is not sufficient to mediate oxygen regulation of $c t a D$, coxll and CycFG expression}

In considering potential molecular explanations for the increased activity of these three promoters at high oxygen tensions, we noted that sequences related to a 

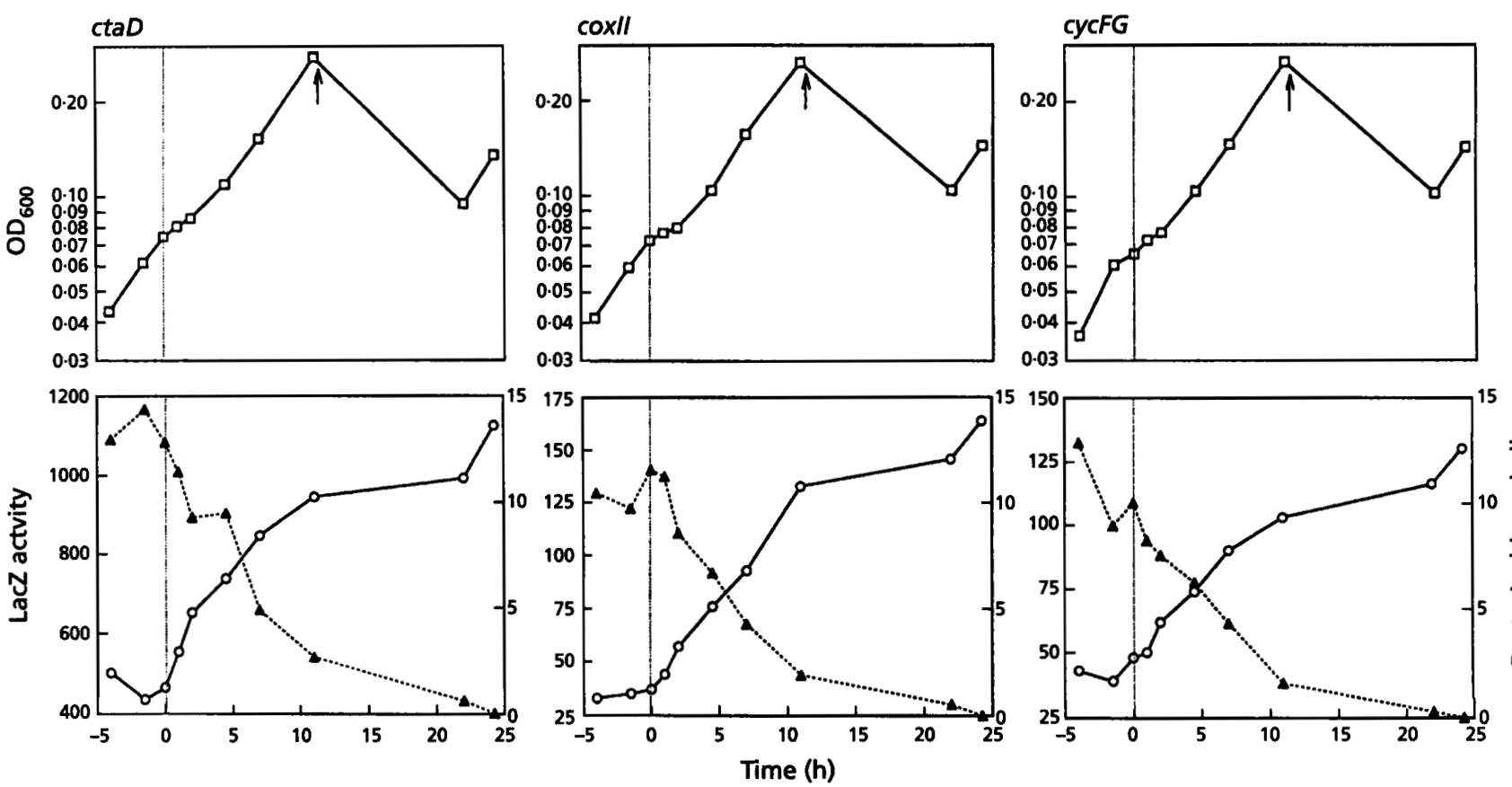

Fig. 4. The effect of oxygen on anaerobic cultures of cells containing individual Lacz operon fusions. Photosynthetic cultures of each strain were shifted to aerobic, dark conditions at time zero (dotted line) and were later diluted to fresh medium for continued aerobic growth (arrow). Top panel: $\square$, culture density $\left(O D_{600}\right)$. Bottom panel: $O$, LacZ activity in Miller units; $\Delta$, specific bacteriochlorophyll content [ $\mu \mathrm{g}$ bacteriochlorophyll (mg protein) ${ }^{-1}$ ].

(a)

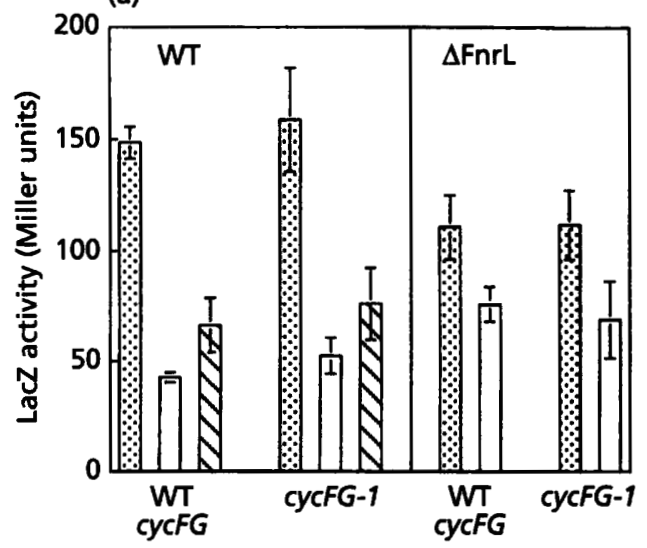

(b)

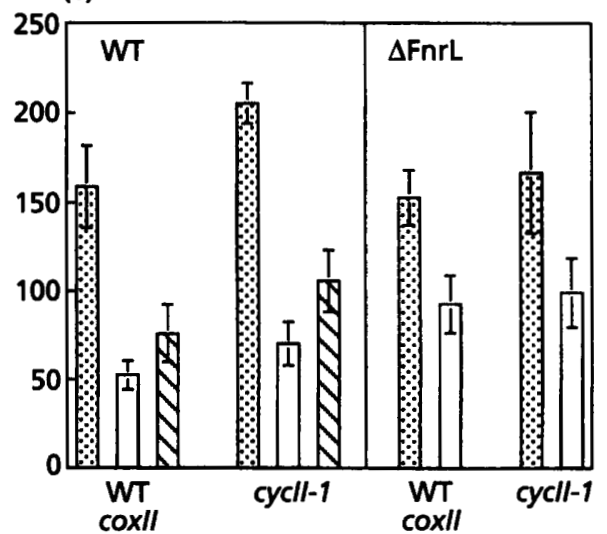

(c)

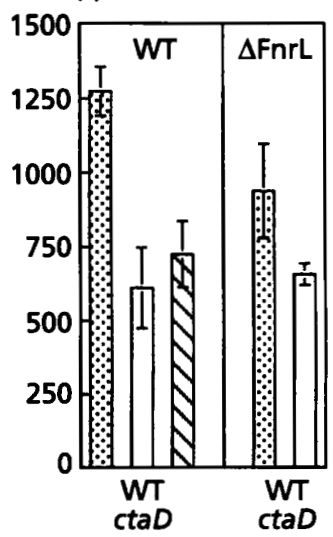

Fig. 5. Effect of mutations in fnrL or the putative FnrL target sites on expression from cycFG, coxll or ctaD reporter genes. LacZ activities of wild-type $R$. sphaeroides $(W T)$ or an FnrL null strain ( $\Delta \mathrm{FnrL}$; JZ1678) containing the indicated reporter plasmids grown under $30 \%$ oxygen (圈), $2 \%$ oxygen $(\square)$ or anaerobic (photosynthetic) $(\mathbb{\Xi}$ ) conditions. Reported values are the mean $\pm S D$ of at least three independent assays and are corrected for activity from the vector alone under the same conditions (pRKK200, <6 Miller units). (a) Wild-type (WT) cycFG::lacZ fusion (plasmid pRKKcycF); cycFG-1::lacZ fusion (plasmid pRKKF-FNR). (b). Wild-type (WT) coxll::lacz fusion (plasmid pRKKcoxII), cycFG-1::lacZ fusion (plasmid pRKKIIFNR). (c). Wild-type ctaD::lacZ fusion (plasmid pRKKctaD).

consensus target site for the $E$. coli global anaerobic transcription factor, FNR, were present upstream of each gene (Fig. 1). While the site in the $c y c F G$ promoter (centred at $-42 \cdot 5$ ) has only $5 / 10$ matches to the $E$. coli FNR consensus, those in the coxII $(-71 \cdot 5)$ and $c t a D$ $(-60.5)$ promoters are identical to this consensus in both half sites. E. coli FNR represses transcription of several aerobic respiratory enzymes (Sharrocks et al., 1991). In the case of the E. coli ndh promoter, FNR represses transcription of this aerobic respiratory enzyme under anaerobic conditions by binding to independent sites centred at -50.5 and -94.5 bp upstream of the start of transcription (Green \& Guest, 1994). Thus, it would not be surprising to find that 


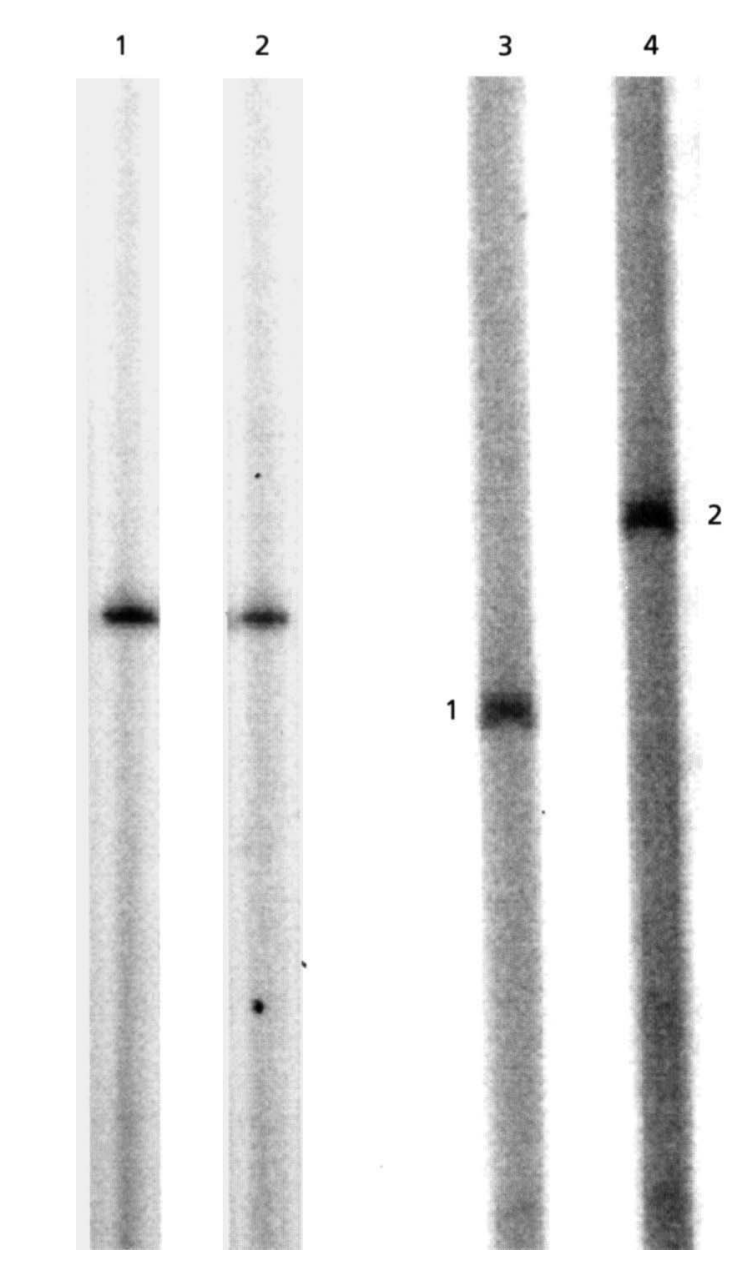

Fig. 6. Mutation of the putative FNR target sites does not alter the $5^{\prime}$ end of coxll and cycFG transcripts. Primer extension assays using a lacZ-specific primer with RNA from cells containing: lane 1, (from left) a wild-type coxll::lacZ fusion (plasmid pRKKcoxll; $15 \mu \mathrm{g}$ RNA); lane 2, a coxll-1::lacZ fusion (plasmid pRKKIIFNR; $15 \mu \mathrm{g}$ RNA); lane 3, a wild-type cycFG::lacZ fusion (plasmid pRKKcycF; $30 \mu \mathrm{g}$ ); or lane 4 , a cycFG-1::lacZ fusion (plasmid pRKKF-FNR; $30 \mu \mathrm{g}$ ) are shown. As described in Methods, the $17 \mathrm{nt}$ difference between cycFG primer extension products 1 and 2 reflects the addition of vector sequences during construction of the cycFG-1::lacZ fusion.

transcription of $c y c F G$ or genes encoding individual subunits of the $R$. sphaeroides cyt $a a_{3}$ were negatively regulated by either the $R$. sphaeroides FNR homologue, FnrL, or a related protein that recognized these sites.

E. coli FNR is believed to make specific contacts at the first, third and fourth nucleotides of the consensus halfsite, TTGAT (Lazazzera et al., 1993; Spiro et al., 1990). As a test of whether $R$. sphaeroides FnrL might repress transcription by binding to regions that are related to the $E$. coli FNR consensus, a single mutant derivative of the $c y c F G$ ( $c y c F G-1)$ and coxII (coxIl-1) promoters was generated in which all of the putative contact points were replaced with non-consensus bases that are known to abolish DNA binding by the E. coli protein (Fig. 1;
Ziegelhoffer \& Kiley, 1995). R. sphaeroides FnrL has considerable amino acid sequence identity with $E$. coli FNR in the putative DNA binding domain, so it is expected that the site-specific protein-DNA contacts would be similar (Zeilstra-Ryalls \& Kaplan, 1995). Within experimental error, LacZ levels from cells containing the mutant reporter genes were indistinguishable from those of cells harbouring a wild-type reporter fusion under all tested conditions (Fig. 5a-c). Thus, it appears that these FNR sites are not essential for the reduced activity of the $c y c F G$ and coxII promoters under low oxygen or anaerobic conditions. Control experiments suggest these mutations did not introduce unwanted secondary effects on promoter function since primer extension assays showed that the transcription start sites from these mutant reporter genes were the same as from their wild-type counterparts (Fig. 6).

As an independent test of the role of FnrL in ctaD, coxll and $c y c F G$ promoter function, LacZ levels produced from wild-type and mutant reporter plasmids were measured in the $R$. sphaeroides FnrL null mutant, JZ1678 (Fig. 5a-c). Because the FnrL null mutant is photosynthetically incompetent (Zeilstra-Ryalls \& Kaplan, 1995), LacZ levels were only measured from cultures grown in the presence of $30 \%$ or $2 \%$ oxygen (a condition which approximates the effects of anaerobic growth on $c t a D$, coxIl and $c y c F G$ promoter function, Fig. 3). At high ( $30 \%$ ) oxygen tensions, LacZ levels from the $c y c F G-1$ (Fig. 5a) and coxII-1 (Fig. 5b) fusions were nearly identical to their wild-type counterparts in both wild-type cells and the FnrL null mutant. Normal activity of the coxII-1 and $c y c F G-1$ mutant promoters in the FnrL null mutant at $30 \%$ oxygen is a clear indication that this global regulator is not functioning under these conditions. Similarly, LacZ levels produced from the coxII- 1 promoter at $2 \%$ oxygen in the $\Delta \mathrm{FnrL}$ mutant were indistinguishable from those measured in cells containing a wild-type coxIl reporter gene (Fig. 5b). This provides an indication that this target site is not solely responsible for reducing function of the coxIl promoter under these conditions. In contrast, there was a slight, but reproducible, increase in activity of the cycFG (Fig. 5a) and coxII (Fig. 5b) promoters (25-30\%) at $2 \%$ oxygen in the FnrL null mutant compared to wild-type cells (Fig. 5). While this reproducible increase in LacZ levels suggests that FnrL has some role in reducing transcription from the $c y c F G$ and coxII promoters at low oxygen tension, the magnitude of the effect is below the twofold increase expected if this protein were the sole negative regulator of these genes under these conditions.

When the above results are considered together, it appears likely that FnrL is not solely responsible for reducing $c t a D$, coxII and $c y c F G$ promoter activity under the conditions we have tested $[2 \%$ oxygen and anaerobic (photosynthetic) growth]. However, it is still possible that FnrL or a related protein contributes to reduced transcription of these $R$. sphaeroides aerobic cytochrome genes under these or other conditions we did not test. For example, action of $E$. coli FNR can often require 


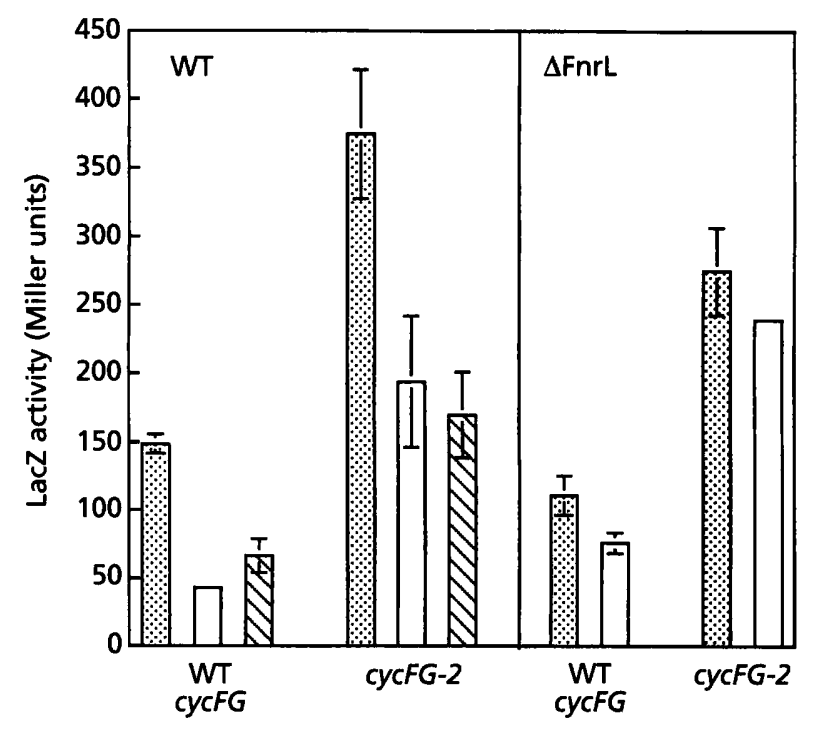

Fig. 7. Effect of the cycFG-2 mutation on promoter activity. LacZ activities of wild-type $R$. sphaeroides 2.4.1 or an FnrL null strain ( $\triangle \mathrm{FnrL} ; \mathrm{JZ1678)}$ containing either a wild-type (WT) cycFG::lacZ fusion (plasmid pRKKcycF) or the cycFG-2::lacZ fusion (plasmid pRKKF-REP) grown under $30 \%$ oxygen (圆), $2 \%$ oxygen $(\square)$ or anaerobic (photosynthetic) ( $\$$ ) conditions are shown. Reported values are the mean \pm SD of at least three independent assays and are corrected for activity from the vector alone under the same conditions (pRKK200, <6 Miller units). Note that under $2 \%$ oxygen conditions, the SO in LacZ levels from wild-type cells carrying the wild-type cycFG plasmid and $\Delta F n r L$ cells carrying the promoter mutant plasmid were each $<5$ Miller units.

additional proteins (Spiro \& Guest, 1991), so the failure of the target site or host mutations to fully increase $c y c F G$, coxII or ctaD transcription at low oxygen tensions could reflect the action of additional transcription factors. E. coli FNR interacts at two sites within the $n d h$ promoter (Green \& Guest, 1994), so it is possible that additional non-consensus FNR sites in these promoters might prevent us from observing a strong effect of the mutations we made. $R$. sphaeroides is also one of a growing number of eubacteria which have been found to contain more than one protein in the FNR family (Cuypers \& Zumft, 1993; Tosques et al., 1996). Thus, the potential for related proteins, such as NnrR from $R$. sphaeroides strain 2.4.3 (Tosques et al., 1996), to alter $c t a D$, coxII or $c y c F G$ promoter function in the presence or absence of FnrL can not be ruled out at this time (if this protein exists in strain 2.4.1).

\section{An inverted repeat overlapping the cycFG promoter is not essential for oxygen regulation}

We also tested whether a previously noted inverted repeat (centred at $-24 \cdot 5$ ) regulated $c y c F G$ promoter function (Flory \& Donohue, 1995). When a mutation that destroyed the downstream half-site (without changing the upstream half-site that overlaps a potential -35 element; Fig. 1) was generated (cycFG-2), LacZ levels from this mutant reporter gene increased approximately twofold in cells grown at $30 \%$ oxygen (Fig. 7). The

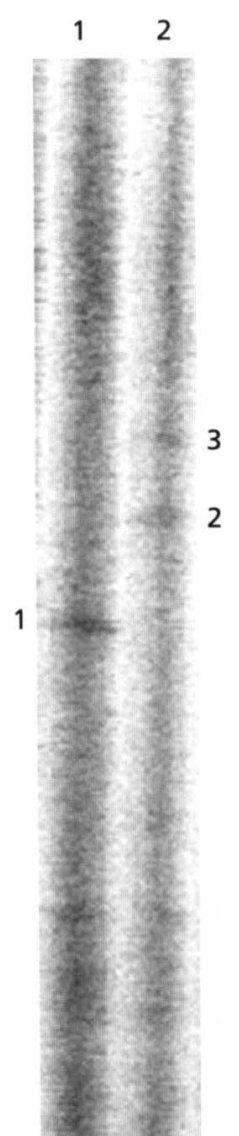

Fig. 8. The cycFG-2 mutation creates a new promoter. Primer extension assays of $20 \mu \mathrm{g}$ RNA from photosynthetically grown wild-type cells containing: lane 1, a wild-type cycFG::lacZ fusion (plasmid pRKKcycF); or lane 2, a cycFG-2::lacZ fusion (plasmid pRKKF-REP) are shown. Primer extension product 1 is the wild-type $5^{\prime}$ end; product 2 depicts the wild-type transcript displaced $17 \mathrm{nt}$ by addition of vector sequence during construction of the cycFG-2 allele (Methods); product 3 denotes the new 5 ' end created by the cycFG-2 allele.

cycFG-2 allele appears to have created a new promoter since these cells contain a second primer extension product that is $14 \mathrm{nt}$ longer than the wild-type transcript (Fig. 8). Even though the $c y c F G-2:$ : lacZ fusion probably contains multiple promoters, transcription under $2 \%$ oxygen and anaerobic conditions was reduced to an extent comparable to its wild-type counterpart. Thus, we can conclude that the downstream half-site is not essential for decreased $c y c F G$ promoter function at low oxygen tensions. Finally, changes in oxygen tension must affect both potential promoters since the abundance of both primer extension products is lowered under photosynthetic conditions (data not shown).

\section{Is oxygen regulation of the coxll, CtaD and CycFG promoters independent of systems which control photosynthesis genes?}

In summary, our data show that transcription of genes for cyt $a a_{3}$ subunits as well as the cycFG operon is reduced at low oxygen tension. The slow, gradual 
increase in $c t a D$, coxII and $c y c F G$ transcription after oxygen is added to photosynthetic cultures contrasts dramatically with the rapid induction of photosynthetic gene expression seen when respiratory cultures of purple bacteria like $R$. sphaeroides are shifted to low oxygen or anaerobic conditions that trigger intracytoplasmic membrane formation (Cohen-Bazire et al., 1957; Chory et al., 1984). Thus, it will be interesting to define the regulators of these aerobic cytochrome genes, explore if these transcription factors interact with proteins or products accumulated under photosynthetic conditions and determine if the similar patterns of $c t a D$, coxII and cycFG promoter function reflects their existence in a regulon of genes with similar metabolic functions.

\section{ACKNOWLEDGEMENTS}

This research was supported by NIH grant GM37509 to T.J.D. During the initial phases of this work, J.E.F. was supported by NIH Biotechnology Training Grant GM08349 to University of Wisconsin-Madison. We thank Sheila Ferguson-Miller and Jim Shapleigh for the cytochrome oxidase genes, Jill Zeilstra-Ryalls and Sam Kaplan for providing the FnrL null mutant prior to publication, Russ Karls for pRKK200 and valuable advice and Patricia Kiley for advice and comments on this manuscript.

\section{REFERENCES}

Bagdasarian, M. \& Timmis, K. N. (1982). Host: vector systems for gene cloning in Pseudomonas. Curr Top Microbiol Immunol 96, 47-67.

Cao, J., Shapleigh, J., Gennis, R., Revzin, A. \& Ferguson-Miller, S. (1991). The gene encoding cytochrome $c$ oxidase subunit II from Rhodobacter sphaeroides; comparison of the deduced amino acid sequences of corresponding peptides from other species. Gene 101, 133-137.

Cao, J., Hosler, J., Shapleigh, J., Revzin, A. \& Ferguson-Miller, S. (1992). Cytochrome $a a_{3}$ of Rhodobacter sphaeroides as a model for mitochondrial cytochrome $c$ oxidase: the coxII/coxIII operon codes for structural and assembly proteins homologous to those in yeast. $J$ Biol Chem 267, 24273-24278.

Chory, J., Donohue, T. J., Varga, A. R., Staehlin, L. A. \& Kaplan, S. (1984). Induction of photosynthetic membranes of Rhodopseudomonas sphaeroides: biochemical and morphological studies. J Bacteriol 159, 540-554.

Cohen-Bazire, G., Sistrom, W. R. \& Stanier, R. Y. (1957). Kinetic studies of pigment synthesis by non-sulfur purple bacteria. J Cell Comp Physiol 49, 25-68.

Cuypers, H. \& Zumft, W. G. (1993). Anaerobic control of denitrification in Pseudomonas stutzeri escapes mutagenesis of an fnr-like gene. J Bacteriol 175, 7236-7246.

Devereux, J., Haeberli, P. \& Smithies, O. (1984). A comprehensive set of sequence analysis programs for the VAX. Nucleic Acids Res 12, 387-395.

Donohue, T. J. \& Kaplan, S. (1991). Genetic techniques in the Rhodospirillaceae. Methods Enzymol 204, 459-485.

Donohue, T. J., McEwan, A. G. \& Kaplan, S. (1986). Cloning, DNA sequence, and expression of the Rhodobacter sphaeroides cytochrome $c_{2}$ gene. J Bacteriol 168, 962-972.

Donohue, T. J., McEwan, A. G., Van Doren, S., Crofts, A. \& Kaplan, S. (1988). Phenotypic and genetic characterization of cytochrome $c_{2}$ deficient mutants of Rhodobacter sphaeroides. Biochemistry 27, 1918-1925.

Eraso, J. M. \& Kaplan, S. (1994). prrA, a putative response regulatory protein involved in oxygen regulation of photosynthesis gene expression in Rhodobacter sphaeroides. J Bacteriol $176,32-43$.

Flory, J. E. \& Donohue, T. J. (1995). Organization and expression of the Rhodobacter sphaeroides cycFG operon. J Bacteriol 177, 4311-4320.

García-Horsman, J. A., Barquera, B., Rumbley, J., Ma, J. \& Gennis, R. B. (1994a). The superfamily of heme-copper respiratory oxidases. J Bacteriol 176, 5587-5600.

García-Horsman, J. A., Berry, E., Shapleigh, J. P., Alben, J. O. \& Gennis, R. B. (1994b). A novel cytochrome $c$ oxidase from Rhodobacter sphaeroides that lacks $\mathrm{Cu}_{\mathrm{A}}$. Biochemistry 33, 3113-3119.

Gomelsky, M. \& Kaplan, S. (1995). Genetic evidence that PpsR from Rhodobacter sphaeroides 2.4.1 functions as a repressor of puc and bchF expression. J Bacteriol 177, 1634-1637.

Green, J. \& Guest, J. R. (1994). Regulation of transcription at the $n d h$ promoter of Escherichia coli by FNR and novel factors. Mol Microbiol 12, 433-444.

luchi, S., Chepuri, V., Fu, H. A., Gennis, R. B. \& Lin, E. C. C. (1990). Requirement for terminal cytochromes in generation of the signal for the arc regulatory system in Escherichia coli: study using deletions and lac fusions to cyo and cyd. J Bacteriol 172, 6020-6025.

Karls, R., Schulz, V., Jovanovich, S. B., Flynn, S., Pak, A. \& Reznikoff, W. S. (1989). Pseudorevertants of a lac promoter mutation reveal overlapping nascent promoters. Nucleic Acids Res 17, 3927-3949.

Kunkel, T. A. (1985). Rapid and efficient site-specific mutagenesis without phenotypic selection. Proc Natl Acad Sci USA 82, 488-492.

Lazazzera, B. A., Bates, D. M. \& Kiley, P. J. (1993). The activity of the Escherichia coli transcription factor FNR is regulated by a change in oligomeric state. Genes Dev 7, 1993-2005.

Lee, J. K. \& Kaplan, S. (1992). cis-acting regulatory elements involved in oxygen and light control of puc operon transcription in Rhodobacter sphaeroides. J Bacteriol 174, 1146-1157.

Markwell, M. A. K., Haas, S. M., Bieber, L. L. \& Tolbert, N. E. (1978). A modification of the Lowry procedure to simplify protein determination in membrane and lipoprotein samples. Anal Biochem 87, 206-210.

Minagawa, J., Nakamura, H., Yamato, I. \& Anraku, Y. (1990). Transcriptional regulation of the cytochrome $b_{562}-O$ complex in Escherichia coli. J Biol Chem 265, 11198-11203.

Moshiri, F., Smith, E. G., Taormino, J. P. \& Maier, R. J. (1991). Transcriptional regulation of cytochrome $d$ in nitrogen-fixing Azotobacter vinelandii. J Biol Chem 266, 23169-23174.

Penfold, R. J. \& Pemberton, J. M. (1994). Sequencing, chromosomal inactivation, and functional expression in Escherichia coli of $p p s R$, a gene which represses carotenoid and bacteriochlorophyll synthesis in Rhodobacter sphaeroides. J Bacteriol 176, 2869-2876.

Prentki, P. \& Krisch, H. M. (1984). In vitro insertional mutagenesis with a selectable DNA fragment. Gene 29, 303-313.

Sambrook, J., Fritsch, E. F. \& Maniatis, T. (1989). Molecular Cloning: a Laboratory Manual, 2nd edn. Cold Spring Harbor, NY: Cold Spring Harbor Laboratory.

Schilke, B. A. \& Donohue, T. J. (1992). $\delta$-Aminolevulinate couples 
$c y c A$ transcription to changes in heme availability in $R$ bodobacter sphaeroides. J Mol Biol 226, 101-115.

Shapleigh, J. P. \& Gennis, R. B. (1992). Cloning, sequencing and deletion from the chromosome of the gene encoding subunit I of the $a a_{3}$-type cytochrome $c$ oxidase of Rhodobacter sphaeroides. Mol Microbiol 6, 635-642.

Sharrocks, A. D., Green, J. \& Guest, J. R. (1991). FNR activates and represses transcription in vitro. Proc $R$ Soc Lond B 245, 219-226.

Simon, R., Priefer, U. \& Puhler, A. (1983). A broad host range mobilization system for in vivo genetic engineering: transposon mutagenesis in Gram-negative bacteria. Bio/Technology 1, 784-791.

Spiro, S. \& Guest, J. R. (1991). Adaptive responses to oxygen limitation in Escherichia coli. Trends Biochem Sci 16, 310-314.

Spiro, S., Gaston, K. L., Bell, A. I., Roberts, R. E., Busby, S. J. W. \& Guest, J. R. (1990). Interconversion of the DNA-binding specificities of two related transcription regulators, CRP and FNR. Mol Microbiol 4, 1831-1838.

Tosques, I. E., Shi, J. \& Shapleigh, J. R. (1996). Cloning and characterization of $n n r R$, whose product is required for the expression of proteins involved in nitric oxide metabolism in Rhodobacter sphaeroides 2.4.3. J Bacteriol 178, 4958-4964.

Trumpower, B. L. \& Gennis, R. B. (1994). Energy transduction by cytochrome complexes in mitochondrial and bacterial respiration: the enzymology of coupling electron transfer reactions to transmembrane proton translocation. Annu Rev Biochem 63, 675-716.

Zeilstra-Ryalls, J. H. \& Kaplan, S. (1995). Aerobic and anaerobic regulation in Rhodobacter sphaeroides 2.4 .1 : the role of the fnrL gene. J Bacteriol 177, 6422-6431.

Zhu, Y. S. \& Kaplan, S. (1985). Effects of light, oxygen, and substrates on steady-state levels of mRNA coding for ribulose1,5-bisphosphate carboxylase and light-harvesting and reaction center polypeptides in Rhodopseudomonas sphaeroides. J Bacteriol 162, 925-932.

Ziegelhoffer, E. \& Kiley, P. J. (1995). In vitro analysis of a constitutively active mutant form of the Escherichia coli global transcription factor FNR. J Mol Biol 245, 351-361.

Received 10 January 1997; revised 8 June 1997; accepted 19 June 1997. 